\title{
Adaptive IEEE 802.15.4 Protocol for Energy Efficient, Reliable and Timely Communications *
}

\author{
Pangun Park \\ ACCESS Linnaeus Center \\ Royal Institute of Technology \\ 100-44, Stockholm, Sweden \\ pgpark@ee.kth.se
}

\author{
Carlo Fischione \\ ACCESS Linnaeus Center \\ Royal Institute of Technology \\ 100-44, Stockholm, Sweden \\ carlofi@ee.kth.se
}

\author{
Karl Henrik Johansson \\ ACCESS Linnaeus Center \\ Royal Institute of Technology \\ 100-44, Stockholm, Sweden \\ kallej@ee.kth.se
}

\begin{abstract}
The IEEE 802.15.4 standard for wireless sensor networks can support energy efficient, reliable, and timely packet transmission by tuning the medium access control parameters macMinBE, macMaxCSMABackoffs, and macMaxFrameRetries. Such a tuning is difficult, because simple and accurate models of the influence of these parameters on the probability of successful packet transmission, packet delay and energy consumption are not available. Moreover, it is not clear how to adapt the parameters to the changes of the network and traffic regimes by algorithms that can run on resourceconstrained nodes. In this paper, an effective analytical model is used to derive an adaptive algorithm at the medium access control layer for minimizing the power consumption while guaranteeing reliability and delay constraints in the packet transmission. The algorithm does not require any modifications of the IEEE 802.15.4 standard and can be easily implemented on existing network nodes. Numerical results show that the analysis is accurate, that the proposed algorithm satisfies reliability and delay constraints, and ensures a longer lifetime of the network under both stationary and transient network conditions.
\end{abstract}

\section{Categories and Subject Descriptors}

C.2.2 [Computer Systems Organization]: Computer-Communication Networks-Network Protocols

\section{General Terms}

Algorithms, Design, Performance, Standardization, Theory.

\section{Keywords}

Wireless sensor network, IEEE 802.15.4, Optimization, Adaptive tuning.

\footnotetext{
${ }^{*}$ This work was supported by the EU project FeedNetBack, the Swedish Research Council, the Swedish Strategic Research Foundation, and the Swedish Governmental Agency for Innovation Systems.
}

Permission to make digital or hard copies of all or part of this work for personal or classroom use is granted without fee provided that copies are not made or distributed for profit or commercial advantage and that copies bear this notice and the full citation on the first page. To copy otherwise, to republish, to post on servers or to redistribute to lists, requires prior specific permission and/or a fee.

IPSN'10, April 12-16, 2010, Stockholm, Sweden.

Copyright 2010 ACM 978-1-60558-988-6/10/04 ...\$10.00.

\section{INTRODUCTION}

The IEEE 802.15.4 [1] standard has received considerable attention as a low data rate and low power protocol for wireless sensor network (WSN) applications in industry, control, home automation, health care, and smart grids $[1,2,3]$. Many of these applications require that packets ${ }^{1}$ are received with a given probability of success. In addition to such a reliability constraint, other applications ask for timely packet delivery [4]. It is known that IEEE 802.15.4 may have poor performance in terms of power consumption, reliability and delay [5], unless the medium access control (MAC) parameters are properly selected. Therefore, it is essential to develop methods to tune the IEEE 802.15.4 MAC parameters to enhance the network lifetime and improve the quality of the service experienced by the applications running on top of the network.

This paper focuses on the optimization of the power consumption with reliability and delay constraints for the IEEE 802.15.4 MAC. We consider the analytical studies of the IEEE 802.15.4 which capture the protocol behavior, where there are retry limits to send packets, acknowledgements (ACKs), and unsaturated traffic. We use this model to pose an optimization problem where the objective function is the power consumption of the network, and the constraints are the reliability and delay of the packet delivery. This mathematical problem is specially appealing for many control and industrial applications [2]. Since high reliability and low delay may demand a significant energy consumption, thus reducing the WSN lifetime [4]-[6], the reliability and delay must be flexible design parameters that need to be adequate for the requirements. Note that controllers can usually tolerate a certain degree of packet losses and delay [7] and [8]. Hence, the maximization of the reliability and minimization of the delay are not the optimal design strategies for the control applications. We especially focus on maximization of the network lifetime by taking into account the tradeoff between energy consumption and application requirements with the dynamic and continuous adaptation of the network operations to the traffic and channel conditions. Our aim is the design of distributed and adaptive algorithms that are simple to implement on sensor nodes, but still flexible, scalable, and able to provide high quality of service for WSN applications. Throughout this paper, we will focus on the MAC layer of the IEEE 802.15.4 standard, whereas we do not deal with the physical layer.

The remainder of this paper is as follows. In Section 2, we summarize existing work of adaptive tuning of the IEEE 802.15.4 MAC protocol. Section 3 lists the main contributions of the paper and their relation to the literature. We describe the slotted carrier sense multiple access with collision avoidance (CSMA/CA) mechanism of the IEEE 802.15.4 standard in Section 4. In Section 5, we

\footnotetext{
${ }^{1}$ Throughout this paper, we refer to packets as medium access control protocol data units, or MAC frames.
} 
present the analytical model of the performance metrics based on a generalized Markov chain model of the slotted CSMA/CA algorithm. In Section 6, the optimization problem to adapt the MAC parameters is investigated. In addition, practical issues on how to implement the algorithm on sensors are also discussed. Numerical results achieved during stationary and transitionary conditions are reported in Section 7. Finally, Section 8 concludes the paper.

\section{RELATED WORK}

Several algorithms to tune the MAC parameters of IEEE 802.11 [9] and IEEE 802.15.4 have been proposed. We consider IEEE 802.11 since the IEEE 802.15.4 MAC is strictly related to IEEE 802.11. The algorithms can be grouped in those based on the use of physical layer measurements, and those based on the use of linklayer information.

An adaptive tuning based on physical layer measurements has been investigated in [10]-[12], where [10] and [12] consider a $p$ persistent approximation of the IEEE 802.11 MAC protocol and [11] applies a similar method for the IEEE 802.15.4 MAC protocol to optimize the average backoff window size. The channel access probability $p$ that maximizes the throughput or minimizes the power consumption is derived. This algorithm and its scalability to the network size have been studied also for IEEE 802.15.4 [11]. However, that study was less successful, because the channel sensing mechanism, the optional acknowledgement (ACK), and retransmission mechanisms are hard to be approximated by a $p$-persistent MAC. Furthermore, in [11] and [12] a saturated traffic regime is assumed, which is a scenario of reduced interest for typical WSN applications.

Link-based optimizations for IEEE 802.11 and 802.15.4 have been investigated in [13]-[17], where simple window adjustment mechanisms that are based on ACK transmissions have been considered. In these papers, the algorithms adapt the contention window size depending on the successful packet transmission, packet collision and channel sensing state, but the algorithms are not grounded on an analytical study. In [13]-[15], a heuristic multiplicative increase linear decrease (MILD) backoff algorithm is applied to improve the fairness of channel usage for IEEE 802.11. In [13], additional control packets and a different backoff algorithm with a backoff copy scheme are used to alleviate fairness problem. It uses a request to send/clear to send (RTS/CTS), synchronization and ACK message exchange. In [14], the fairness index is defined by incorporating both the per-node and per-link fairness. Each node adjusts its contention window to maximize the fairness. A similar self-adaptive backoff algorithm is investigated to improve the channel throughput and the fairness of channel usage in [15]. A linkbased algorithm of the IEEE 802.15.4 random backoff mechanism to maximize the throughput has been presented in [16]. In [17], a dynamic tuning algorithm of the contention window size is evaluated on goodput, reliability, and average delay.

Any IEEE 802.15.4 enhancement based on the use of link-layer information has some drawback. First, it requires a modification of the standard. Then, although link-based mechanisms are simple to implement, the ACK mechanism may be costly since it introduces large overhead for small packets. For instance, alarm messages in industrial control application are a single byte whereas the ACK has a size of 11 bytes. In addition, the ACK mechanism requires extra waiting time. Moreover, link-based algorithms adapt the MAC parameters for each received ACK, which leads to a slow and inefficient adaptation to network, traffic, and channel variations under low traffic scenarios.

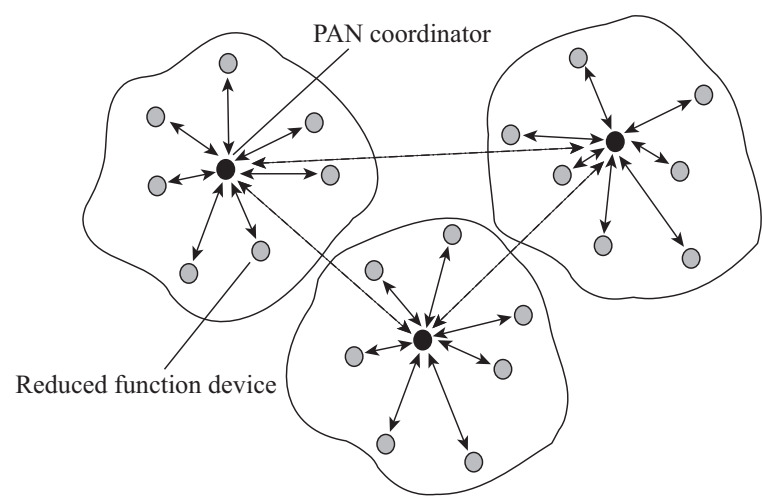

Figure 1: Star network topology of IEEE 802.15.4. The packets generated by the nodes (grey circle) are transmitted toward the PAN coordinator (black circle) depicted in the middle of each cluster.

\section{ORIGINAL CONTRIBUTION}

We consider a star network with a personal area network (PAN) coordinator and $N$ nodes as in Fig. 1 . The nodes use a beaconenabled slotted CSMA/CA with ACKs. The important parameters of the IEEE 802.15.4 CSMA/CA algorithm are the minimum value of the backoff exponent macMinBE, the maximum number of backoffs macMaxCSMABackoffs, and the maximum number of retries macMaxFrameRetries. We describe the CSMA/CA algorithm of IEEE 802.15.4 in Section 4.

In this paper, we propose a new adaptive tuning of the IEEE 802.15.4 MAC protocol for reliable and timely communication while minimizing the energy consumption. The protocol is adjusted dynamically by a constrained optimization problem. The objective function, denoted by $\widetilde{E}_{\text {tot }}$, is the total energy consumption of a node for transmitting packets plus the energy for receiving the ACK at the node. The constraints are given by the probability of successful packet delivery (reliability) and average delay that such a transmitting node experiences. The constrained optimization problem that every generic transmitting node in the network solves is

$$
\begin{array}{ll}
\min _{\mathbf{V}} & \widetilde{E}_{\text {tot }}(\mathbf{V}) \\
\text { s.t. } & \widetilde{R}(\mathbf{V}) \geq R_{\min }, \\
& \widetilde{D}(\mathbf{V}) \leq D_{\max }, \\
& \mathbf{V}_{0} \leq \mathbf{V} \leq \mathbf{V}_{m} .
\end{array}
$$

The decision variables $\mathbf{V}=\left(m_{0}, m, n\right)$ are

$$
\begin{aligned}
m_{0} & \triangleq \text { macMinBE }, \\
m & \triangleq \text { macMaxCSMABackoffs }, \\
n & \triangleq \text { macMaxFrameRetries } .
\end{aligned}
$$

$\widetilde{R}(\mathbf{V})$ is the reliability, and $R_{\min }$ is the minimum desired probability for successful packet delivery. $\widetilde{D}(\mathbf{V})$ is the average delay for a successfully received packet, and $D_{\max }$ is the desired maximum average delay. The constraint $\mathbf{V}_{0} \leq \mathbf{V} \leq \mathbf{V}_{m}$ captures the limited range of the MAC parameters. In the problem, we used the symbol $\sim$ to evidence that the energy, reliability, and delay expression are approximations. We will show later that we use approximations of high accuracy and reduced computational complexity. Notice also that the energy, reliability, and delay expressions are implicit function of the traffic and of the network topology. This is due to 
that the busy channel probability and the collision probability experienced by the IEEE 802.15.4 MAC, which are components of the reliability and delay, are function of the traffic and the network topology, as we will see later.

We remark here that the adaptive scheme proposed in this paper does not require information coming from the ACK as opposed to link-layer based adaptation mechanisms. This is because the ACK would provide a slow feedback to implement an adaptation, whereas our adaptive scheme is based on the estimation of the busy channel probabilities. This information is available on a much shorter time scale then the ACK information. Moreover, the use of the ACK in link-layer based adaptation requires a modification of the backoff mechanism of the standard. By contrast, we do not impose any modification of the standard in this paper.

Main contributions of the paper are the following: (a) formulation and solution of a novel optimization problem for the MAC parameters, (b) discussion on a practical implementation of the optimization by an adaptive algorithm and (c) performance evaluations of the algorithm by simulation of both stationary and transient network conditions.

In [18], a simplified and effective model is proposed for the IEEE 802.15.4 MAC. It considers a generalized Markov model of the exponential backoff process including retry limits, acknowledgements and unsaturated traffic regime. We modify this model to reduce drastically the computational complexity while ensuring a satisfactory accuracy. Based on this modelling, we propose an adaptive tuning of MAC parameters that uses the physical layer measurement of the channel sensing. This adaptive IEEE 802.15.4 MAC is furnished with two distinctive features: it does not require any modifications of the existing standard, and it makes a global optimization of the MAC parameters. Specifically, in contrast to link-based adaptation [15]-[17], our algorithm estimates the the busy channel probability and the channel access probability as input variables, which does not require an ACK mechanism or RTS/CTS handshakes (or related modification of the IEEE standard). In contrast to [10]-[12], we do not use the (inaccurate) $p$-persistent approximation and the modification of the standard therein proposed, and we do not require any hardware modification to estimate the signal-to-noise ratio. Our adaptive tuning optimizes the considered MAC parameters all at once, and not only some of them, as proposed in [10]-[17].

The proposed adaptive IEEE 802.15.4 MAC improves the power efficiency substantially while guaranteeing reliability and delay constraints. The adaptation is achieved by distributed asynchronous iterations that only require channel condition information, the number of nodes of the network, and the traffic load. We show that the algorithm convergence is fast and is robust to errors in the estimation of the channel condition, number of nodes, and traffic load. A good fairness is also achieved.

\section{OVERVIEW OF THE IEEE 802.15.4}

The standard defines two channel access modalities: the beaconenabled modality, which uses a slotted CSMA/CA and exponential backoff, and a simpler unslotted CSMA/CA without beacons.

Consider a node trying to transmit. In the slotted CSMA/CA variant of IEEE 802.15.4, first the MAC sub-layer of the node initializes four variables, i.e., the number of backoffs $(\mathrm{NB}=0)$, contention window $(\mathrm{CW}=2)$, backoff exponent $(\mathrm{BE}=$ macMinBE $)$, and retransmission times $(\mathrm{RT}=0)$. Then the MAC sub-layer delays for a random number of complete backoff periods in the range $\left[0,2^{\mathrm{BE}}-\right.$ 1] units. When the backoff period is zero, the node performs the first clear channel assessment (CCA). If two consecutive CCAs are idle, then the node commences the packet transmission. If ei- ther of the CCA fails due to a busy channel, the MAC sublayer will increase the value of both $\mathrm{NB}$ and $\mathrm{BE}$ by one up to a maximum value macMaxCSMABackoffs and macMaxBE, respectively. Hence, the value of $\mathrm{NB}$ and $\mathrm{BE}$ depend on the number of $\mathrm{CCA}$ failures of a packet. Once the BE reaches macMaxBE, it remains at the value of macMaxBE until it is reset. If NB exceeds macMaxCSMABackoffs, then the packet is discarded due to the channel access failure. Otherwise the CSMA/CA algorithm generates a random number of complete backoff periods and repeat the process. Here, the variable macMaxCSMABackoffs represents the maximum number of times the CSMA/CA algorithm is required to backoff. If channel access is successful, the node transmits the frame and waits for ACK. The reception of the corresponding ACK is interpreted as successful packet transmission. If the node fails to receive ACK due to collision or ACK timeout, the variable RT is increased by one unit up to macMaxFrameRetries units. If RT is less than macMaxFrameRetries, the MAC sublayer initializes two variables $\mathrm{CW}=0, \mathrm{BE}=$ macMinBE and follows the CSMA/CA mechanism to re-access the channel. Otherwise the packet is discarded due to the retry limits.

By knowing the duration of an ACK frame, ACK timeout, interframe spacing (IFS), data packet length, and header duration, we define the packet successful transmission time $L_{s}$ and the packet collision time $L_{c}$ as

$$
\begin{aligned}
& L_{s}=L+t_{\mathrm{ack}}+L_{\mathrm{ack}}+I F S, \\
& L_{c}=L+t_{\mathrm{m}, \mathrm{ack}},
\end{aligned}
$$

where $L$ is the total length of a packet including overhead and payload, $t_{\text {ack }}$ is ACK waiting time, $L_{\text {ack }}$ is the length of ACK frame, and $t_{\mathrm{m}, \text { ack }}$ is the timeout of the ACK, see details in [1].

In the following section, we analyze the reliability, delay and power consumption of IEEE 802.15.4 exponential backoff process.

\section{ANALYTICAL MODELLING}

In a star network, all $N$ nodes contend to send data to the PAN coordinator, which is the data sink in Fig. 1. We assume no hidden node. Throughout this paper we consider applications where nodes asynchronously generate packets with probability $1-q$, when a node sends a packet successfully, discard a packet or the sampling interval is expired. Otherwise a node stays for $L_{0} S_{b} \mathrm{~s}$ without generating packets with probability $q$, where $L_{0}$ is an integer and $S_{b}$ is the time unit aUnitBackoffPeriod (corresponding to 20 symbols). The data packet transmission is successful if an ACK packet is received. In addition, we assume that the channel is sensed busy or idle without errors.

In such a scenario, a precise and effective analytical model of the slotted CSMA/CA by a Markov chain model was proposed in [18]. The precise model gives us the objective function, energy (1a), and constraints on reliability (1b) and delay (1c) of the optimization problem in a numerical form. These expressions are a function of the busy probability $\alpha$ of the first clear channel assessment $\left(\mathrm{CCA}_{1}\right)$, the busy probability $\beta$ of the second clear channel assessment $\left(\mathrm{CCA}_{2}\right)$, and the probability $\tau$ that a node attempts $\mathrm{CCA}_{1}$. Note that the probabilities $\alpha, \beta, \tau$ are assumed to be independent. The expressions of $\tau, \alpha$, and $\beta$ are derived by solving numerically a system of non-linear equations, as it is common in the related literature, see, e.g., $[5,19,20]$. Note that $\alpha$ and $\beta$ typically take quite different values, as it was shown in in [5].

The drawback of the accurate analysis given in [18] is that there is no closed form expression for the carrier sensing probability and the busy channel probabilities, since the system of equations that gives $\tau, \alpha$ and $\beta$ must be solved by numerical methods. This may 


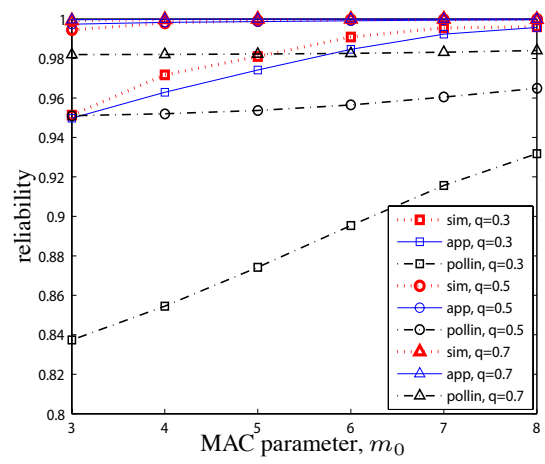

(a) $m_{0}=3, \ldots, 8, m_{b}=8, m=4, n=3$

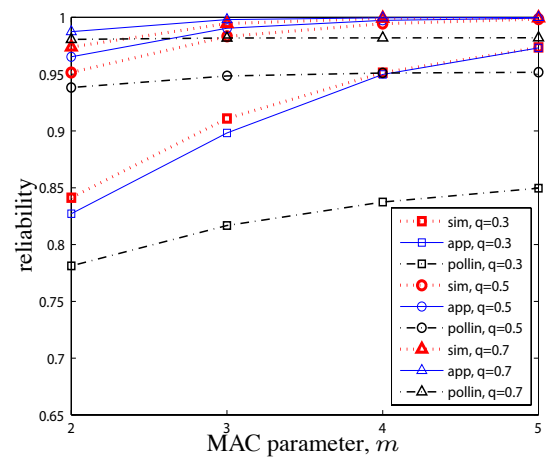

(b) $m=2, \ldots, 5, m_{0}=3, m_{b}=8, n=3$

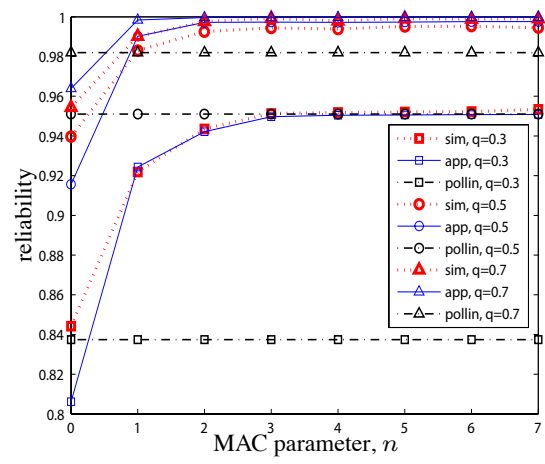

(c) $n=0, \ldots, 7, m_{0}=3, m_{b}=8, m=4$

Figure 2: Reliability as a function of the traffic regimes $q=0.3,0.5,0.7$, and MAC parameters $m_{0}=3, \ldots, 8, m_{b}=8, m=2, \ldots, 5$, $n=0, \ldots, 7$ with Pollin's Markov chain model. The length of the packet is $L=0.96 \mathrm{~ms}$ and the number of nodes is $N=20$.

be computationally demanding and therefore inadequate for use in simple sensor devices. Here, we present an approximated model that is a modification of the analysis proposed in [18] to reduce the computation complexity for optimization purposes on sensor nodes. The key idea is that sensor nodes can estimate the busy channel probabilities and the channel sensing probability. Specifically, we propose complete closed form expressions by reducing the complexity of the expressions proposed in [18], and we model the wake-up behavior of nodes. Moreover, nodes exploit local measurements to evaluate the performance metrics, rather than solving nonlinear equations. In the following, we present the analytical expressions for the energy consumption, reliability, and delay. Monte Carlo simulations validate the proposed models.

\subsection{Reliability}

The main contributions of this section is the approximated expression of the reliability (1b) of the optimization problem (1), where we recall that the reliability is the probability of successful packet reception. We have the following result:

APPROXImATION 1. An approximation of the reliability is

$$
\widetilde{R}(\boldsymbol{V})=1-x^{m+1}(1+\widetilde{y})-\widetilde{y}^{n+1}
$$

where

$$
\begin{gathered}
\widetilde{y}=\left(1-\left(1-(1+x)(1+\hat{y}) \widetilde{b}_{0,0,0}\right)^{N-1}\right)\left(1-x^{2}\right), \\
\widetilde{b}_{0,0,0}=\frac{2}{2^{m_{0}} r_{1}+2 r_{2}},
\end{gathered}
$$

where

$$
\begin{aligned}
& r_{1}=(1+2 x)(1+\hat{y}), \\
& r_{2}=L_{s}\left(1-x^{2}\right)(1+\hat{y})+\frac{L_{0} q\left(1+\hat{y}^{2}+\hat{y}^{n+1}\right)}{1-q}, \\
& \hat{y}=\left(1-(1-\tau)^{N-1}\right)\left(1-x^{2}\right),
\end{aligned}
$$

and $x=\alpha+(1-\alpha) \beta$.

Proof. A proof is given in [21].

We note that the reliability derived above is a function of channel measurements $\alpha, \beta$, and $\tau$, and MAC parameters $m_{0}, m$, and $n$, and the traffic condition $q$ and $L_{0}$. Note that as the MAC parameters $m_{0}, m$, and $n$ increase the reliability increases. In addition, as the traffic decreases $q \rightarrow 1$ or $L_{0} \rightarrow \infty$, the probability $\widetilde{b}_{0,0,0}$ increases and this causes an increase of the reliability. The basic idea for the derivation of this expression of the reliability is that, in slotted CSMA/CA, packets are unsuccessfully received due to two reasons: channel access failure and retry limits. Channel access failure happens when a packet fails to obtain idle channel in two consecutive CCAs within $m+1$ backoffs. Furthermore, a packet is discarded if the transmission fails due to repeated collisions after $n+1$ attempts.

We use Monte Carlo simulations of the slotted CSMA/CA algorithm [1] to validate the approximated model of the reliability given by Eq. (3). The simulations are based on the specifications of the IEEE 802.15.4 with several values of the traffic regime and MAC parameters. Simulation data was collected out of 5 runs, each lasting $2 \times 10^{5}$ time slots. Fig. 2 compares Eq. (3), the analytical model in [5], and Monte Carlo simulations as a function of the traffic regimes $q=0.3,0.5,0.7$ with $N=20$ nodes and different MAC parameters $m_{0}, m, n$. In the figure, note that "Pollin" refers to the reliability model derived in [5]. Our analytical expression matches quite well the simulation results. The expression is closer to simulation results under low traffic regime $q=0.5,0.7$ than high traffic regime $q=0.3$. The reliability approaches 1 under very low traffic regime $q=0.7$. In Fig. 2(a), 2(b), the reliability increases as MAC parameters $m_{0}, m$ increase, respectively. In Fig. 2(c), we observe that the improvement of reliability is small as the retry limits $n$ increases for $n \geq 3$. Notice that the reliability saturates to 0.95 for traffic regime $q=0.3$ for $n \geq 3$. Hence, the retransmissions are necessary but not sufficient to obtain high reliability under high traffic regimes.

\subsection{Delay}

The average delay for a successfully received packet is defined as the time interval from the instant the packet is at the head of its MAC queue and ready to be transmitted, until the transmission is successful and the ACK is received.

Approximation 2. The expected value of the approximated delay is

$$
\begin{aligned}
\widetilde{D}(\boldsymbol{V})= & T_{s}+\mathbb{E}\left[\widetilde{T}_{h}\right] \\
& +\left(\frac{y}{1-y}-\frac{(n+1) y^{n+1}}{1-y^{n+1}}\right)\left(T_{c}+\mathbb{E}\left[\widetilde{T}_{h}\right]\right) .
\end{aligned}
$$

where $y=\left(1-(1-\tau)^{N-1}\right)\left(1-x^{m+1}\right)$ and the expected value 


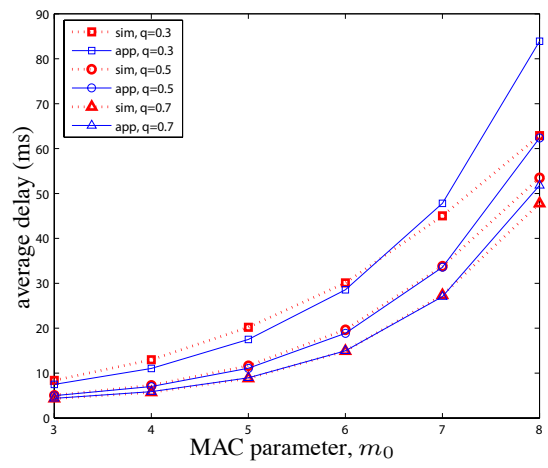

(a) $m_{0}=3, \ldots, 8, m_{b}=8, m=4, n=3$

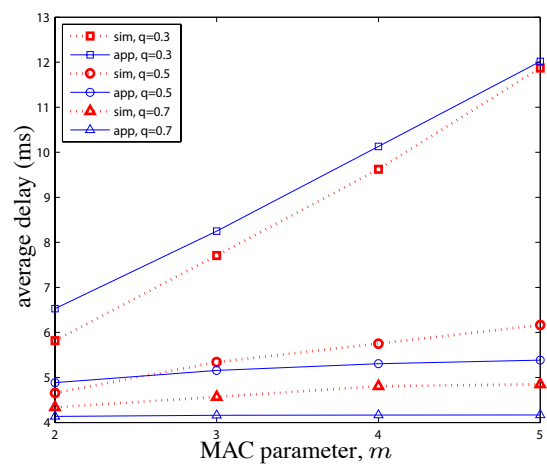

(b) $m=2, \ldots, 5, m_{0}=3, m_{b}=8, n=3$

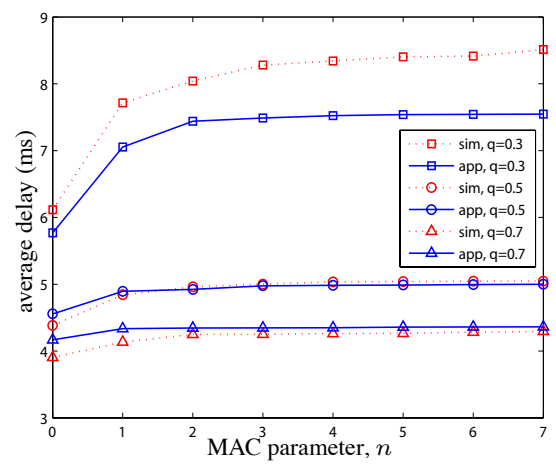

(c) $n=0, \ldots, 7, m_{0}=3, m_{b}=8, m=4$

Figure 3: Average delay as a function of the traffic regimes $q=0.3,0.5,0.7$ and MAC parameters $m_{0}=3, \ldots, 8, m_{b}=8$, $m=2, \ldots, 5, n=0, \ldots, 7$. The length of the packet is $L=0.96 \mathrm{~ms}$ and the number of nodes is $N=20$.

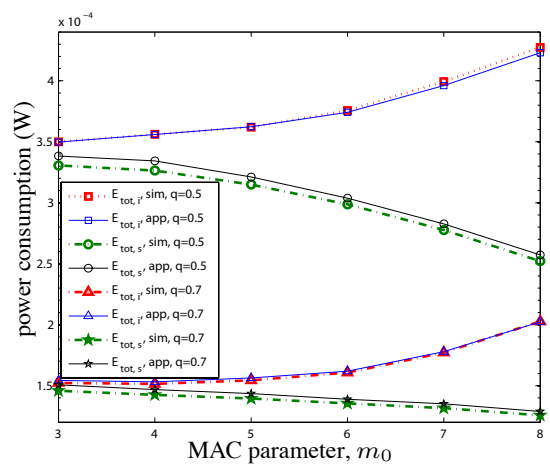

(a) $m_{0}=3, \ldots, 8, m_{b}=8, m=4, n=3$

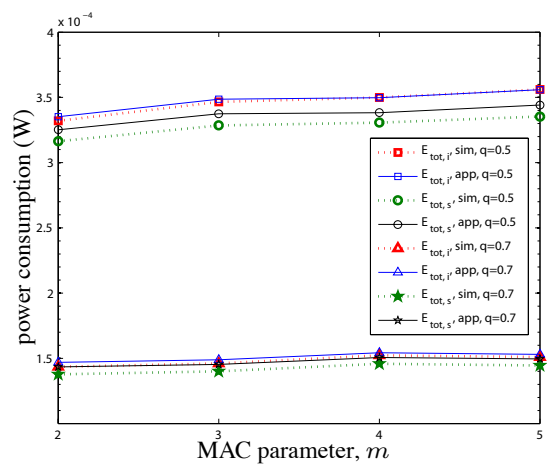

(b) $m=2, \ldots, 5, m_{0}=3, m_{b}=8, n=3$

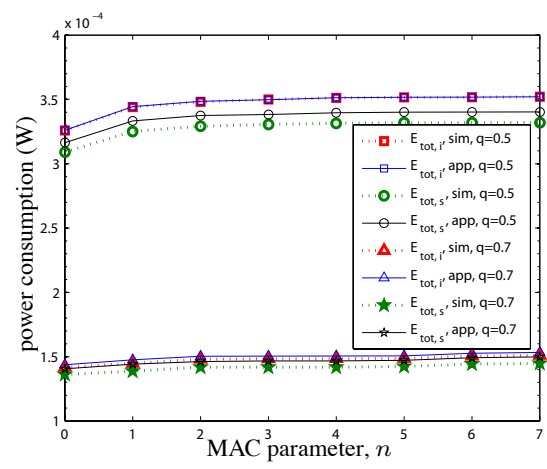

(c) $n=0, \ldots, 7, m_{0}=3, m_{b}=8, m=4$

Figure 4: Average power consumption of $\boldsymbol{I}$-mode and $\boldsymbol{S}$-mode as a function of the traffic regimes $q=0.3,0.5,0.7$ and $\mathrm{MAC}$ parameters $m_{0}=3, \ldots, 8, m_{b}=8, m=2, \ldots, 5, n=0, \ldots, 7$. The length of the packet is $L=0.96$ ms and the number of nodes is $N=20$.

of the approximated backoff delay is

$$
\begin{aligned}
\mathbb{E}\left[\widetilde{T}_{h}\right]= & 2 S_{b}\left(1+\frac{1}{4}\left(\frac { 1 - \gamma } { 1 - \gamma ^ { m + 1 } } \left(2^{m_{0}+1} \frac{1-(2 \gamma)^{m+1}}{1-2 \gamma}\right.\right.\right. \\
& \left.\left.\left.-\frac{3(m+1) \gamma^{m+1}}{1-\gamma}\right)+\frac{3 \gamma}{1-\gamma}-\left(2^{m_{0}}+1\right)\right)\right),
\end{aligned}
$$

and $\gamma=\max (\alpha,(1-\alpha) \beta)$.

Proof. A proof is given in [21].

The average delay derived above is a function of channel estimations $\alpha, \beta$, and $\tau$ and MAC parameters $m_{0}, m, n$. In Eq. (5), the first term $T_{s}+\mathbb{E}\left[\widetilde{T}_{h}\right]$ represents the average packet delay for a successfully received packet without any collisions and the second term takes into account the extra delay due to packet collision. Recall that $L_{s}$ is the time for successfully transmitted packets and $L_{c}$ is the packet collision time given in Eq. (2). The backoff delay $\mathbb{E}\left[\widetilde{T}_{h}\right]$ is due to the busy channel sensing during $\mathrm{CCA}_{1}$ and $\mathrm{CCA}_{2}$. Hence, there are two main reasons of packet delay: delay due to packet collision and backoff delay due to busy channel.

Fig. 3 shows the average delay as obtained by Eq. (5) as a function of different traffic regimes $q=0.3,0.5,0.7$ with a given number of nodes $N=20$ and different MAC parameters $m_{0}, m$ and $n$. The analytical model predicts well the simulation results. The accuracy is reduced under high traffic regime $q=0.3$ due to the approximation. Observe that the average delay increases as traffic regime increases due to high busy channel probability and collision probability. Fig. 3(a) shows that the average delay increases exponentially as $m_{0}$ increases. Hence, we conclude that $m_{0}$ is the key parameter on average delay in comparison to $m, n$.

\subsection{Power Consumption}

In this section we propose two novel models for the average power consumption that we need in the optimization problem (1). The models depends on the radio state during the backoff mechanism specified by the IEEE 802.15.4 standard. Let us denote by $I$-mode and $S$-mode the situation when the radio is set in idle mode or in sleep mode during backoff period, respectively.

ApProximation 3. The energy consumption of the I-mode is

$$
\begin{aligned}
\widetilde{E}_{\mathrm{tot}, \mathrm{i}}(\boldsymbol{V})= & \frac{P_{i} \tau}{2}\left[\frac{(1-x)\left(1-(2 x)^{m+1}\right)}{(1-2 x)\left(1-x^{m+1}\right)} W_{0}-1\right] \\
& +P_{s c}(2-\alpha) \tau+(1-\alpha)(1-\beta) \tau \\
& \times\left(P_{t} L+P_{i}+L_{\text {ack }}\left(P_{r}\left(1-P_{c}\right)+P_{i} P_{c}\right)\right) \\
& +P_{w} q\left(x^{m+1}(1+y)+P_{c}\left(1-x^{2}\right) y^{n}\right. \\
& \left.+\left(1-P_{c}\right)\left(1-x^{2}\right)(1+y)\right) \widetilde{b}_{0,0,0}
\end{aligned}
$$


and of the S-mode is

$$
\begin{aligned}
\widetilde{E}_{\mathrm{tot}, \mathrm{s}}(\boldsymbol{V})= & P_{s c}(2-\alpha) \tau+(1-\alpha)(1-\beta) \tau \\
& \times\left(P_{t} L+P_{i}+L_{\mathrm{ack}}\left(P_{r}\left(1-P_{c}\right)+P_{i} P_{c}\right)\right) \\
& +P_{w}\left(\tau-\frac{\widetilde{b}_{0,0,0}\left(1-(0.5 x)^{m+1}\right)}{W_{0}(1-0.5 x)} \frac{1-y^{n+1}}{1-y}\right),
\end{aligned}
$$

where the state probability $\widetilde{b}_{0,0,0}$ is given in Eq. (4), the collision probability is $P_{c}=1-(1-\tau)^{N-1}, P_{i}, P_{s c}, P_{s p}, P_{w}, P_{t}$ and $P_{r}$ are the average power consumption in idle-listen, channel sensing, sleep states, wake-up state, transmit and receiving states, respectively.

PROOF. A proof is derived in [21].

In the power consumption of I-mode given in Eq. (6), the first, second, third and fourth term represent the power consumption of backoff, channel sensing, packet transmission and wake-up state when a node generates packets, respectively. Note that the radio state during backoff of I-mode is set in idle mode. In the power consumption of $S$-mode given in Eq. (7), the first, second and third term takes into account the power consumption of channel sensing, packet transmission and wake-up state when the backoff counter is expired, respectively. Node needs to wake-up when the backoff counter is zero since the radio state during backoff of $S$-mode is set in sleep mode.

Fig. 4 compares the analytical model and simulation results of power consumption for both I-mode and S-mode as a function of different traffic regimes $q=0.5,0.7$ with a number of nodes $N=$ 20 and different MAC parameters $m_{0}, m$ and $n$. Note that we list the power consumption of different operation modes in Table I. We observe that the power consumption of I-mode increases as the MAC parameters $\left(m_{0}, m, n\right)$ increase under low traffic regime $q=$ $0.5,0.7$ since the node needs to stay more time in idle sleep stage without packet generation under low traffic regime $q=0.5,0.7$, the main component of average power consumption is the idle backoff time rather than transmit or receiving power consumption. However, the power consumption of $S$-mode decreases as $m_{0}$ increases because of sleep mode during the backoff time. We observe that the power consumption has a weaker dependence on $m$ and $n$ than $m_{0}$.

\section{IEEE 802.15.4 OPTIMIZATION}

In the previous sections we developed the expressions of the performance metrics. Here, we present a novel approach where each node locally solves the optimization problem. Consider the reliability, delay and power consumption as investigated in Section 5. The optimization problem (1) can be written by using Eq. (3) of Approximation 1 for the reliability constraint, Eq. (5) of Approximation 2 for the delay constraint and Eq. (6) or (7) of Approximation 3 for the power consumption. Note that the power consumption is given by Eq. (6) if the I-mode is selected, and it is given by Eq. (7), if the $S$-mode is selected. The solution of the optimization problem gives the optimal MAC parameter $\left(m_{0}^{*}, m^{*}, n^{*}\right)$ that each node uses to minimize its energy expenditure, subject to reliability and delay constraints. Notice that the problem is combinatorial because the decision variables take on discrete values.

A vector of decision variables $\mathbf{V}$ is feasible if the reliability and delay constraints are satisfied. The optimal solution may be obtained by checking every combination of the elements of $\mathbf{V}$ that gives feasibility, and then checking the combination that gives the minimum objective function. Clearly, this approach may have a

\begin{tabular}{|c|c|}
\hline Operation mode & Power consumption \\
\hline$P_{r}, P_{s c}$ & $35.46 \mathrm{~mW}$ \\
\hline$P_{t}$ & $31.32 \mathrm{~mW}$ \\
\hline$P_{w}$ & $54 \mathrm{~mW}$ \\
\hline$P_{i}$ & $0.657 \mathrm{~mW}$ \\
\hline$P_{s p}$ & $0.18 \mu \mathrm{W}$ \\
\hline
\end{tabular}

Table 1: Power consumption of different operation modes.

high computational complexity, since there are $6 \times 4 \times 8=192$ combinations of MAC parameters to check [1]. Therefore, in the following we propose an algorithm that gives the optimal solution by checking just a reduced number of combinations.

From Figs. 2, 3 and 4, we remark here that the reliability and power consumption of both $I$-mode and $S$-mode are increasing function as the parameter $n$ increases. This property is quite useful to solve (1) by a simple algorithm with reduced computational complexity, as we see next.

The search of optimal MAC parameters uses an iterative procedure according to the component-based method [22]. In particular, the probabilities $\alpha, \beta$, and $\tau$ are estimated periodically by each node. If a node detects a change of these probabilities, then the node solves the local optimization problem (1) using these estimated values. The solution is achieved by finding the value of $n$ that minimizes the energy consumption given a pair of values for $m_{0}$ and $m$. Since the power consumption is increasing with $n$, it follows that the minimum is attained at the lowest value of $n$ that satisfies the constraints. Given that the reliability is increasing with $n$, simple algebraic passages give that such a value is $n=f\left(m_{0}, m\right)$, with

$$
f\left(m_{0}, m\right)=\left\lceil\frac{\ln \left(1-x^{m+1}(1+\widetilde{y})-R_{\min }\right)}{\ln (\widetilde{y})}-1\right\rceil,
$$

where $\widetilde{y}=\left(1-(1-\widetilde{\tau})^{N-1}\right)\left(1-x^{2}\right)$ and

$$
\widetilde{\tau}=\frac{2 r_{3}}{2^{m_{0}} r_{1}+2 r_{2}},
$$

with

$$
\begin{aligned}
& r_{1}=(1+2 x)(1+\hat{y}), \\
& r_{2}=L_{s}\left(1-x^{2}\right)(1+\hat{y})+\frac{L_{0} q\left(1+\hat{y}^{2}+\hat{y}^{n+1}\right)}{1-q}, \\
& r_{3}=(1+x)(1+\hat{y}),
\end{aligned}
$$

and $\hat{y}=\left(1-(1-\tau)^{N-1}\right)\left(1-x^{2}\right)$. Eq. (8) returns the optimal retry limits given a pair $m_{0}, m$. Notice that $x$ and $\hat{y}$ are measurable since the node estimates $\alpha, \beta$, and $\tau$. By using Algorithm 1, a node checks just $6 \times 4=24$ combinations of the MAC parameters $m_{0}, m$ instead of $6 \times 4 \times 8=192$ combinations that would be required by an exhaustive search.

We have seen by the Approximations 1,2 and 3 that the performance metrics are a function of the busy channel probabilities $\alpha$ and $\beta$ and the channel access probability $\tau$. Once these probabilities are known at a node, the optimal MAC parameters of that node can be readily computed by Algorithm 1. In the algorithm, the number of nodes and packet generation rates are assumed to be known, whereas the busy channel probability and channel access probability are periodically estimated in each node during the sensing states of the MAC layer, and they do not require an ACK mechanism, as we detail in what follows. In addition, the robustness of the algorithm to possible errors in the estimation of the number of nodes and traffic load is then investigated in Section 7.3. 


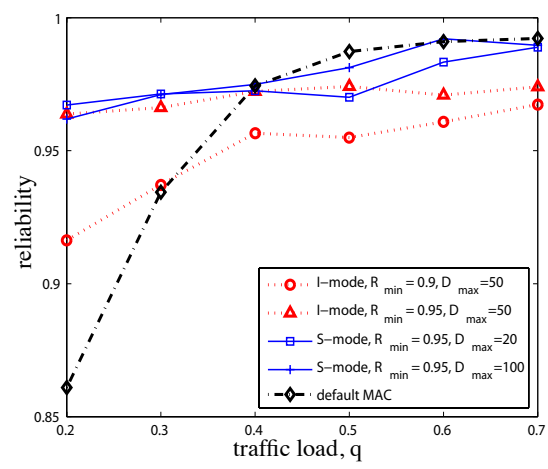

(a) Reliability

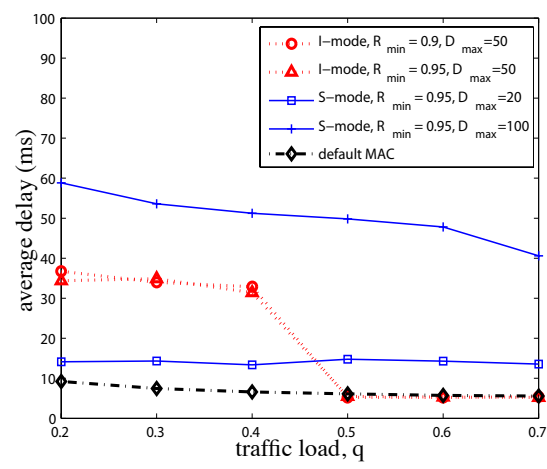

(b) Average delay

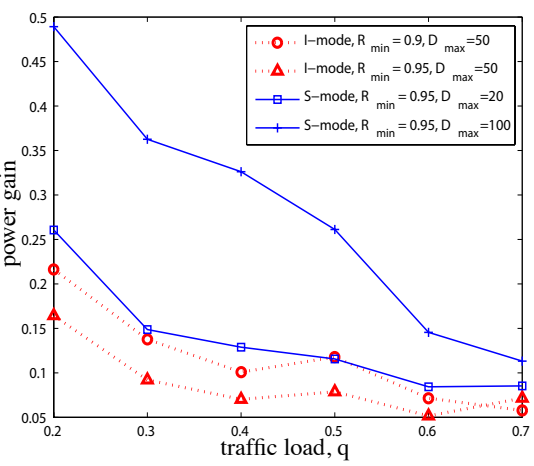

(c) Power gain

Figure 5: Stationary condition: reliability, average delay and power gain of the I-mode, S-mode of proposed scheme and IEEE 802.15.4 with default parameter (macMinBE $=3$, macMaxBE $=5$, macMaxCSMABackoffs $=4$, macMaxFrameRetries $=3$ ) as a function of the traffic load $q=0.2, \ldots, 0.7$, the reliability requirement $R_{\min }=0.9,0.95$ and delay requirement $D_{\text {max }}=$ $20,50,100 \mathrm{~ms}$ for the length of the packet $L=2.2 \mathrm{~ms}$ and $N=10$ nodes. Note that "default MAC" refers to IEEE 802.15.4 with default MAC parameters.

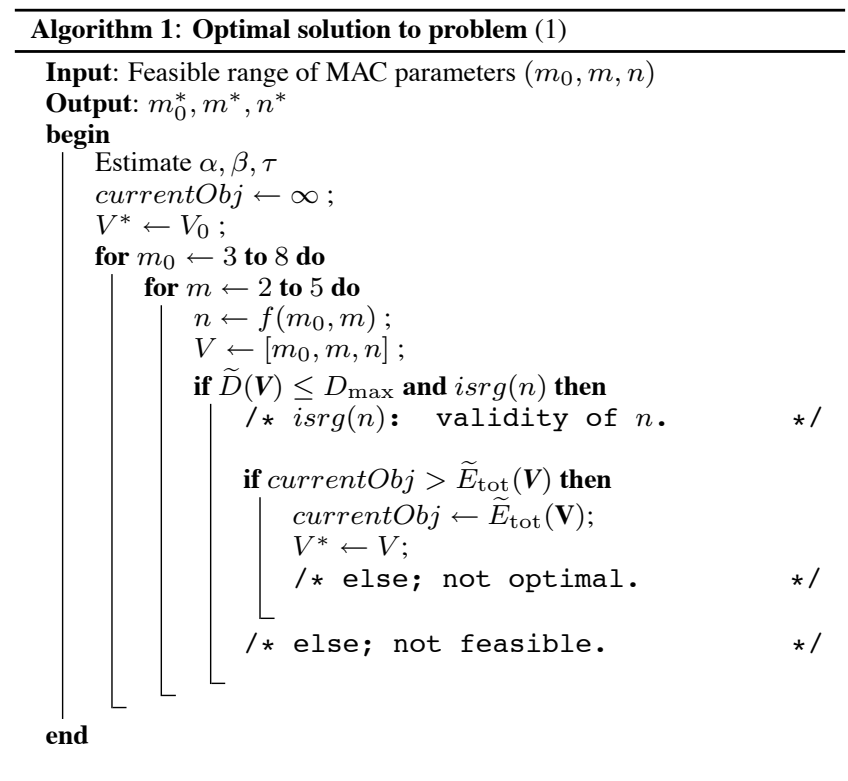

The average busy channel probabilities $\alpha$ and $\beta$ are estimated at each node while sending a data packet to the coordinator. These probabilities are initialized at the beginning of the node's operation. There are several distributed estimation algorithms for wireless sensor networks, see, e.g., [23, 24]. In this paper, we assume that the estimations of the busy channel probabilities and the channel access probability uses a simple first order filter. When the node senses the channel at $\mathrm{CCA}_{1}$ or $\mathrm{CCA}_{2}$, these probabilities are updated using the following recursions

$$
\begin{gathered}
\alpha_{k+1}=\delta_{b} \alpha_{k}+\left(1-\delta_{b}\right) \hat{\alpha}_{k}, \\
\beta_{k+1}=\delta_{b} \beta_{k}+\left(1-\delta_{b}\right) \hat{\beta}_{k},
\end{gathered}
$$

where $k$ denotes the update step for some $\delta_{b} \in(0,1)$, respectively. Note that $\hat{\alpha}$ and $\hat{\beta}$ are the busy channel probability measurements of $\mathrm{CCA}_{1}$ and $\mathrm{CCA}_{2}$, respectively. Each node only counts the number of busy channel events during $\mathrm{CCA}_{1}$ and $\mathrm{CCA}_{2}$ state to obtain $\hat{\alpha}$ and $\hat{\beta}$, respectively. Therefore, a node does not require any ex- tra communication and sensing state to estimate these probabilities compared to the IEEE 802.15.4 standard. By contrast, the estimation algorithms for IEEE 802.11 proposed in [10] and [25] are not energy efficient since a node needs to sense the channel state during the backoff stage. This allows one to estimate the average length of idle period. Hence, these schemes are implementable only in $I$-mode. By contrast, our scheme is applied in both I-mode and $S$ mode and does not require any computation load during the backoff stage. An analysis of the impact of parameter estimation errors is investigated in Section 7.3.

During an initialization phase of the algorithm, a node communicates with the initial MAC parameters $m_{0}=3, m_{b}=8, m=$ $4, n=3$. Then, the busy channel probabilities $\alpha$ and $\beta$ and the channel access probability $\tau$ are estimated in each node during the channel sensing state of IEEE 802.15.4 without any extra states. The application requirements are communicated by the coordinator to the node if there are changes. It is also possible that each node makes a decision of application requirements depending on the data type e.g., strict delay requirement for alarm messages.

\section{NUMERICAL RESULTS FOR THE ADAP- TIVE IEEE 802.15.4 MAC ALGORITHM}

In the following, we present Monte Carlo simulations to analyze the performance of our adaptive tuning algorithm of the MAC parameters, under both stationary and transient conditions. We remark that the analytical modelling that we have proposed in Section 5 is based on a Markov chain which has been validated in [20]. This means that the Monte Carlo simulations that we use here are representative of the real-world behavior of the network.

In the stationary conditions, the application requirements and network scenario are constant, whereas in transient condition there are variations. The simulations are based on the specifications of the IEEE 802.15.4 standard and the practical implementation aspects described in Section 6. In the simulations, the network considers the I-mode and $S$-mode of the node to compare the performance on the reliability, average packet delay and power consumption. Furthermore, we investigate the fairness of resource allocation, robustness to network changes and sensitivity to inaccurate parameter estimations. Note that it is not possible to compare our algorithm to other algorithms from the literature as the link-based ones [15]-[17], because they modify the IEEE 802.15.4 standard 


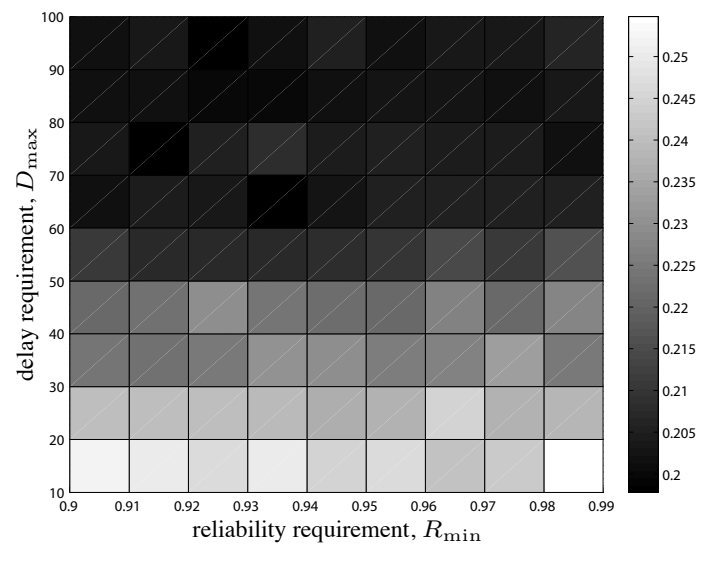

Figure 6: Stationary network condition: power consumption of $\boldsymbol{S}$-mode as a function of reliability constraint $R_{\min }=$ $0.9, \ldots, 0.99$ and delay requirement $D_{\max }=10, \ldots, 100 \mathbf{m s}$ for the traffic load $q=0.5$, the length of packet $L=0.96 \mathrm{~ms}$ and $N=10$ nodes.

and are focused on different performance metrics (e.g., throughput). However, it is possible to show that our algorithm outperforms significantly the results in [15]-[17], since these results are based on the use of ACK feedback, which has a low update frequency with respect to the channel and network variations. Details follow in the sequel.

\subsection{Protocol Behavior in Stationary Conditions}

In this subsection, we are interested in the improvement of performance metrics of the proposed scheme at stationary conditions of the network, namely without changing application requirements and network scenarios. We also present a fairness analysis of the adaptive protocol. Simulation data was collected out of 5 runs, each lasting $2 \times 10^{5}$ time slots.

Figs. 5 compare the reliability, average delay and power gain values of the protocol as obtained by our algorithm and with default MAC parameters. Both the I-mode and $S$-mode for various traffic configurations and requirements are considered. The requirements for both the I-mode and $S$-mode are $R_{\min }=0.9,0.95, D_{\max }=50$ and $R_{\min }=0.95, D_{\max }=20,100 \mathrm{~ms}$, respectively. Fig. 5(a) shows that both $I$-mode and $S$-mode satisfy the reliability constraint for different traffic regimes. We observe strong dependence of the reliability of the default MAC protocol with different traffic regime due to the fixed MAC parameters. At the high traffic regime $q=0.2$, the reliability of default MAC is 0.86. In Fig. 5(b), the delay constraint is fulfilled both I-mode and S-mode. Observe that average delay of I-mode decreases when traffic regime is low $q \geq 0.5$. This is due to that the optimal MAC parameters at higher traffic regime increase more than the ones at lower traffic regime to satisfy the reliability constraint.

Recall that the target of our proposed adaptive algorithm is to use the tradeoff between application constraints and energy consumption instead of just maximization of reliability or minimization of delay. Therefore, to characterize quantitatively the power consumption, we define the power gain as

$$
\rho=\frac{E_{\mathrm{def}}-E_{\mathrm{tot}}(\mathbf{V})}{E_{\mathrm{def}}}
$$

where $E_{\text {def }}$ and $E_{\text {tot }}(\mathbf{V})$ are the average power consumption of

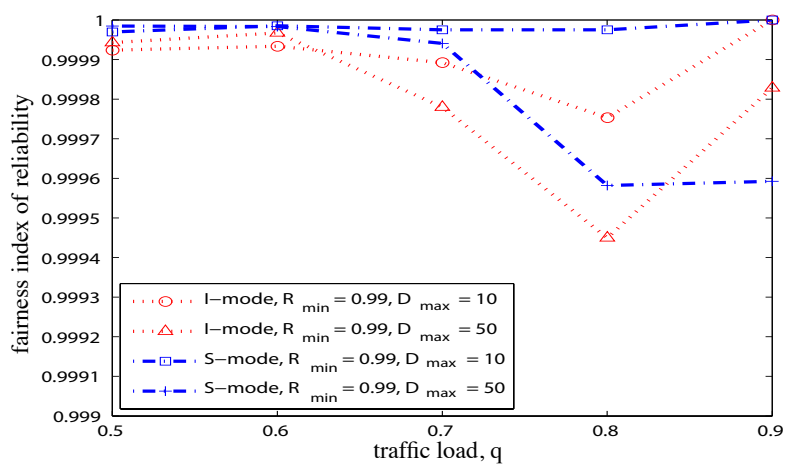

Figure 7: Fairness index of the reliability as a function of the traffic load $q=0.5, \ldots, 0.9$, reliability requirement $R_{\min }=$ 0.99 and delay requirement $D_{\max }=10,50 \mathrm{~ms}$ for the length of the packet $L=0.96 \mathrm{~ms}$ and $N=10$ nodes.

I-mode or $S$-mode for default MAC and proposed scheme, respectively. The closer $\rho$ is to 1 , the better power efficiency. Fig. 5(c) shows that the power gain increases as traffic load increases. This improvement is higher for $S$-mode than $I$-mode, e.g., power gain $\rho \approx 0.49$ for $S$-mode with $R_{\min }=0.95, D_{\max }=100$. Although there is a strong dependence of the power gain on the traffic regime, our proposed algorithm gives a better energy efficiency than the default MAC. Therefore, the numerical results show clearly the effectiveness of our adaptive IEEE 802.15.4 MAC protocol while guaranteeing the constraints.

Next, we observe the tradeoff between the power consumption, reliability and delay constraints. Fig. 6 shows the dependence of the power consumption in $S$-mode with reliability and delay constraints for a given traffic load, packet length, and number of nodes. Observe that as the delay constraint becomes strict the power consumption increases. In other words, the reliability constraint of $S$ mode is less critical than delay constraint, see more results in [21].

The fairness of resource management is one of the most important concerns when implementing the tuning algorithm of the MAC parameters. We use Jain's fairness index [26] to show the fairness of our proposed scheme for both I-mode and S-mode. We compute the fairness index of 10 nodes in a stable network. The closer fairness index to 1 , the better the achieved fairness. Fig. 7 shows the fairness index of the reliability for the different requirements and traffic configurations with a given length of the packet and number of nodes. Fig. 7 reports a very high fairness achievement on reliability greater than 0.999 . A similar behavior is found for delay and power consumption. In other words, the MAC parameters of each node converge to the optimal MAC parameter values. Therefore we conclude that most of the nodes can share equally the common medium.

\subsection{Protocol Behavior in Transient Conditions}

The adaptive IEEE 802.15.4 MAC protocol is based on the estimation of the busy channel probabilities $\alpha$ and $\beta$ and the channel access probability $\tau$. In this section, we investigate the convergence time of the optimal MAC parameters obtained by our adaptive algorithm when the delay constraint changes.

Figs. 8(a), 8(b), 8(c), 8(d) show the behavior of channel state, MAC parameters, reliability and packet delay when the delay requirement changes for both I-mode and $S$-mode with a given traffic load, length of packets, and number of nodes, respectively. Fig. 8(a) reports the busy channel probabilities $\alpha$ and $\beta$ and channel access probability $\tau$ over time. In Section 6, we noticed that the update fre- 


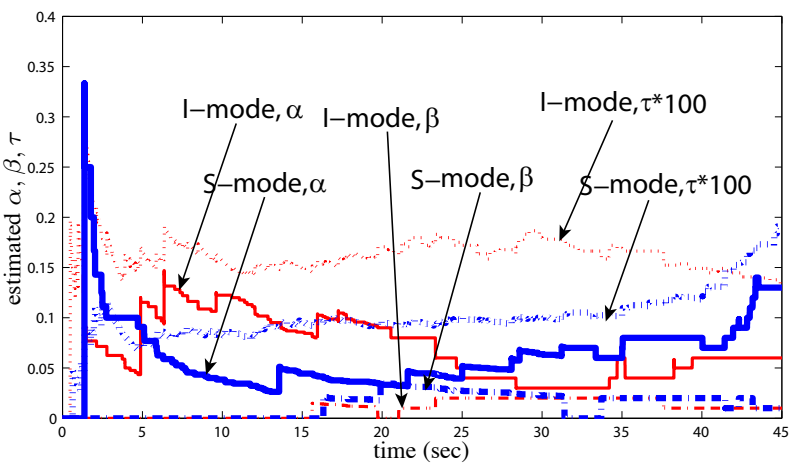

(a) $\alpha, \beta, \tau$ behavior

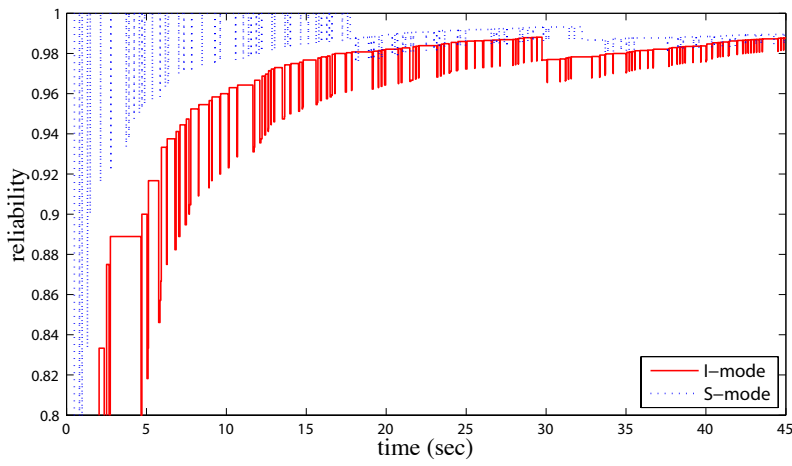

(c) Reliability behavior

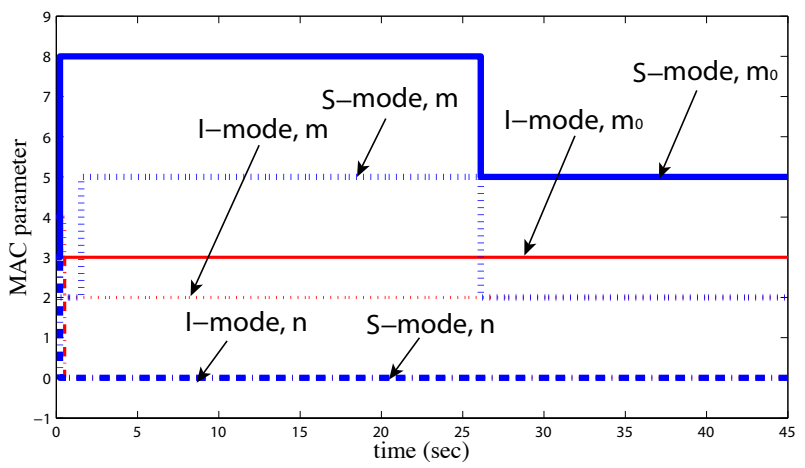

(b) MAC parameter $\left(m_{0}, m, n\right)$ behavior

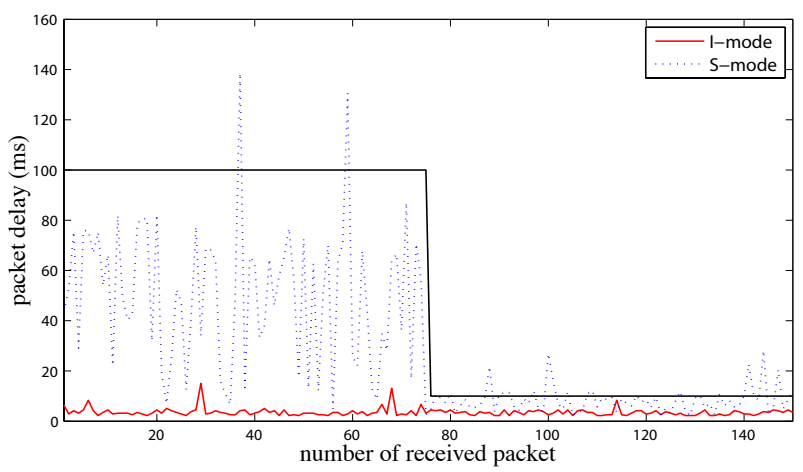

(d) Packet delay behavior

Figure 8: Transient condition: busy channel probabilities, channel access probability, MAC parameters, reliability and delay of I-mode and $\boldsymbol{S}$-mode for the traffic load $q=0.6$, length of the packet $L=0.96 \mathrm{~ms}$ and $N=10$ nodes when the delay requirement changes from $D_{\max }=100 \mathbf{m s}$ to $D_{\max }=10 \mathbf{m s}$ at $26 \mathbf{s}$.

quency of $\alpha, \beta, \tau$ is different. $\tau$ is updated in each aUnitBackoffPer$i o d$ and $\alpha$ and $\beta$ are updated when a node stays in $\mathrm{CCA}_{1}$ and $\mathrm{CCA}_{2}$, respectively. Hence, the update frequency order of $\alpha, \beta$, and $\tau$ is $\tau$ first, then $\alpha$, and finally $\beta$. We remark here that the update frequency of link-based adaptation is lower than the update frequency of $\beta$ of our algorithm since link-based adaptation requires an ACK transmission [15]-[17]. The update frequency of channel estimation is a critical issue where the traffic load is low such as in monitoring applications.

Fig. 8(b) shows the adaptation of the MAC parameters. The optimal $\left(m_{0}, m, n\right)$ of $I$-mode and $S$-mode adapts to $(3,2,0)$ and $(8,5,0)$ before the requirement changes, respectively. Observe that the algorithm returns different parameters for I-mode and $S$-mode due to the different power consumption model, see details in Section 5. After the requirement changes at time $26 \mathrm{~s}$, the MAC parameters $\left(m_{0}, m, n\right)$ of $S$-mode adapt from $(8,5,0)$ to $(5,2,0)$. We observe that the convergence of the MAC parameters of proposed scheme is very fast since our algorithm is based on analytical model instead of heuristic considerations as in link-based adaptation, where the algorithms adapt the contention window size by the ACK transmission [15]-[17]. In addition, recall that our adaptive IEEE 802.15.4 MAC is based on the physical sensing information before transmitting packets.

Fig. 8(c) shows the cumulative packet reception rate of I-mode and $S$-mode. Note that the oscillation of reliability is due to packet loss. In Fig. 8(c), the reliability of $S$-mode is larger than I-mode since the MAC parameters $m_{0}$ and $m$ are larger than the ones of I-mode before the requirement changes. By the same argument, we observe that the packet delay of $S$-mode is about six times the one measured of I-mode in Fig. 8(d). In addition, the packet delay is much more variable in $S$-mode than the one in I-mode. Specifically, with $I$-mode, we have a reduction in the average MAC delay and a shorter tail for the MAC delay distribution with respect to the $S$ mode. After the requirement changes, the packet delay converges to around $10 \mathrm{~ms}$. In addition, the reliability decreases due to the decreasing of the parameters $m_{0}$ and $m$ in Fig. 8(c).

\subsection{Robustness and Sensitivity Analysis}

The performance analysis carried out so far assumed that the number of nodes and traffic configuration are fixed. This assumption has allowed us to verify the effectiveness of our adaptive algorithm for IEEE 802.15.4 MAC in steady state conditions. However, one of the critical issues in the design of wireless networks is time varying conditions. Therefore, in the following analysis, we will investigate how our algorithm reacts to changes in the number of nodes and traffic load when each node has an erroneous estimation of these parameters.

Fig. 9 shows the dynamical behavior of nodes using the I-mode when the number of nodes changes from $N=10$ to $N=20$ with an erroneous estimation of the number of nodes. At time $17.6 \mathrm{~s}$, the number of nodes sharply increases to 20 , when it was estimated to be 10 . We assume that the wrong estimation happens due to some errors in the estimation phase or a biasing induced by the hidden-node phenomenon. This causes a significant increase of the contention level. Note that $n_{1}$ is one of existing nodes before the network change and $n_{11}$ is one of the new nodes that enters the network at time $17.6 \mathrm{~s}$ using its initial MAC parameters. In Fig. 9(a), we observe that the busy channel and channel access probabilities 


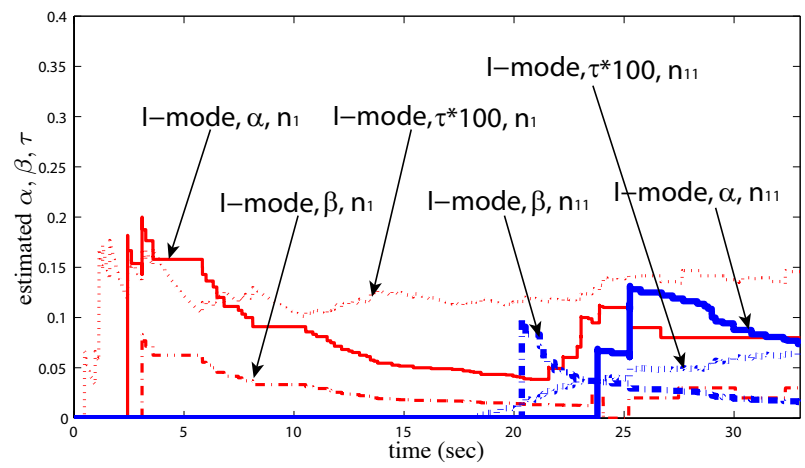

(a) $\alpha, \beta, \tau$ behavior

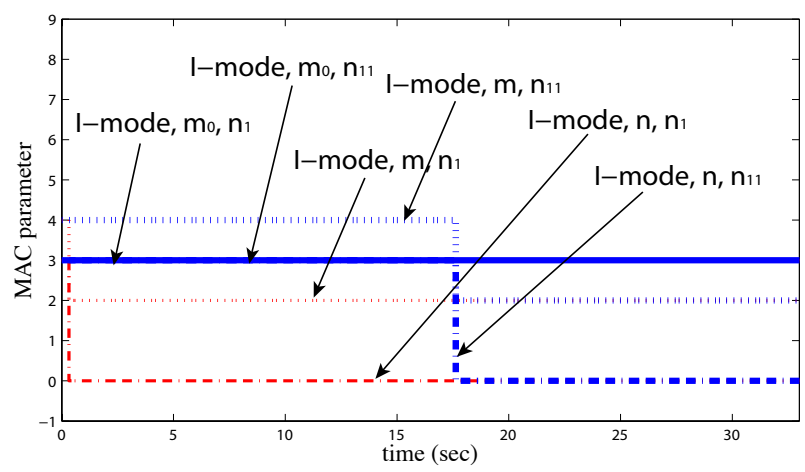

(b) MAC parameter $\left(m_{0}, m, n\right)$ behavior

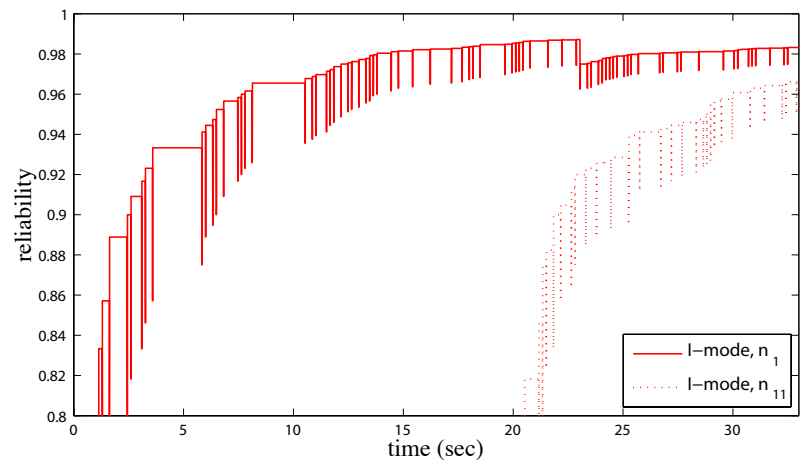

(c) Reliability behavior

Figure 9: Robustness when the number of nodes changes: busy channel probabilities, channel access probability, MAC parameters and reliability behavior of I-mode when the number of nodes changes sharply from $N=10$ to $N=20$ at time $17.6 \mathrm{~s}$ for traffic load $q=0.6$, length of the packet $L=0.96 \mathrm{~ms}$, the reliability and delay constraint $R_{\min }=0.95$ and $D_{\max }=$ $100 \mathrm{~ms}$, respectively. Note that $n_{1}$ and $n_{11}$ represent the behavior of one of $N=10$ nodes plus new nodes after time $17.6 \mathrm{~s}$, respectively.

of node $n_{11}$ become stable after the network changes by updating the MAC parameters. Fig. 9(b) shows that the MAC parameters $\left(m_{0}, m, n\right)$ converge to $(3,2,0)$ for node $n_{1}$ and $n_{11}$. The figures indicate that the system reacts correctly to the erroneous estimation of the number of nodes after a few seconds. In Fig. 9(c), the reliability fulfills the requirement $R_{\min }=0.95$ for both the existing and new nodes. Similar behaviors are observed for $S$-mode, see further details in [21].

Figs. 10 present the behavior of the node when the traffic load changes sharply from $q=0.8$ to $q=0.5$ at time $25.6 \mathrm{~s}$. Nodes use

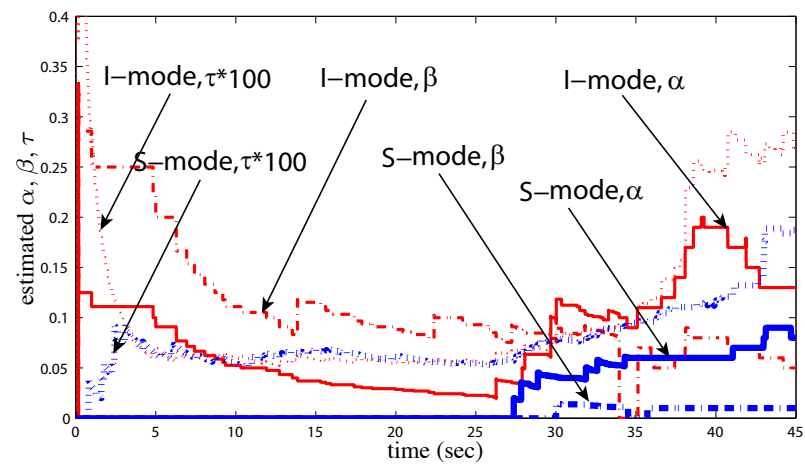

(a) $\alpha, \beta, \tau$ behavior

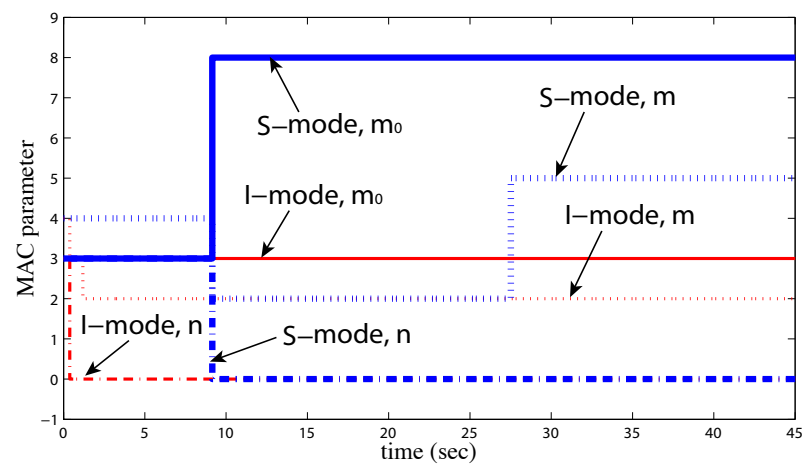

(b) MAC parameter $\left(m_{0}, m, n\right)$ behavior

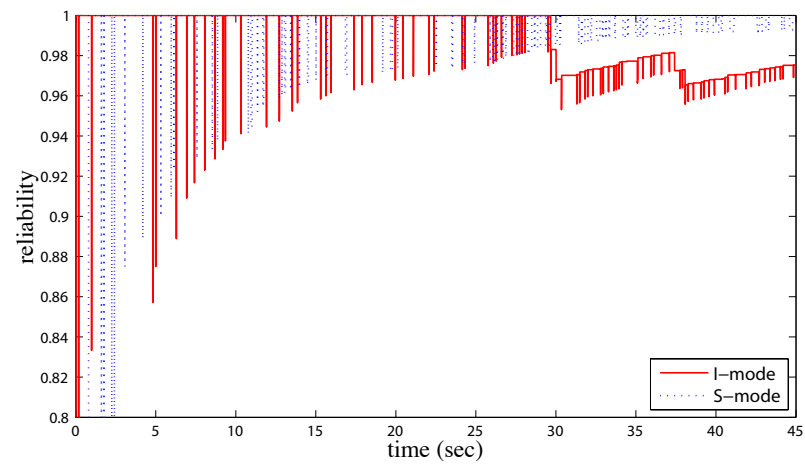

(c) Reliability behavior

Figure 10: Robustness when the traffic load changes: busy channel probabilities, channel access probability, MAC parameters, reliability and delay behavior of $I$-mode and $S$-mode when the traffic load changes sharply from $q=0.8$ to $q=0.5$ at time $25.6 \mathrm{~s}$. The length of the packet is $L=0.96 \mathrm{~ms}$, the reliability and delay constraint are $R_{\min }=0.95$ and $D_{\max }=100 \mathrm{~ms}$, respectively.

a wrong estimation of the traffic load, which is estimated to be $q=0.8$, after the traffic load changes. The results indicate that our algorithm is quite effective for the traffic configuration change. In Fig. 10(a), the busy channel and channel access probability increase as a result of higher traffic regime $q=0.5$ for both $I$-mode and $S$-mode. Fig. 10(b) shows that the parameter $m$ of $S$-mode updates from 2 to 5 due to the increasing busy channel probability after the traffic load changes at time $28 \mathrm{~s}$. The figure indicates that the system reacts correctly to the erroneous estimation of traffic configuration and, in few seconds, the estimation of $\alpha, \beta$ and $\tau$ allow to reach the optimal MAC parameters. In Fig. 10(c), the re- 


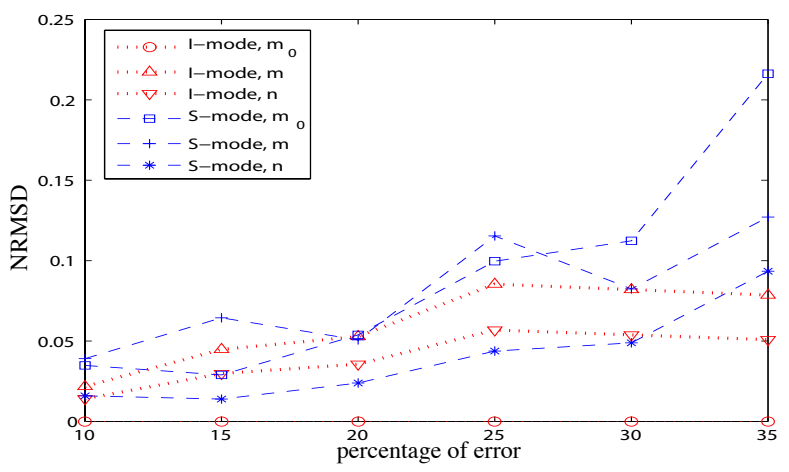

Figure 11: Sensitivity: NRMSD of I-mode and $S$-mode when the traffic load $q=0.6$, length of the packet $L=0.96 \mathrm{~ms}$, reliability requirement $R_{\min }=0.95$ and delay requirement $D_{\max }=100 \mathrm{~ms}$, and $N=10$ nodes with different percentage error in busy channel probabilities $\alpha$ and $\beta$ and channel access probability $\tau$.

liability requirement $R_{\min }=0.95$ is fulfilled for both I-mode and $S$-mode. The reliability of $I$-mode is greater than 0.95 with some fluctuations after the traffic load increases.

In Section 5, we assume that the ideal channel sensing capability of hardware without hidden node terminals. However, this assumption may be not practical due to the hardware failure and time varying wireless condition. Hence, it is important analyze the sensitivity of our adaptive IEEE 802.15.4 MAC to the estimation errors. Fig. 11 illustrates the sensitivity of the proposed scheme with respect to the estimation errors to the busy channel probabilities $\alpha$ and $\beta$ and the channel access probability $\tau$. The normalized root mean squared deviation (NRMSD) between the optimal MAC parameters with exact estimation and the ones with erroneous estimation is used as the indicator of sensitivity. The normalization is taken over the range of MAC parameters $\left(m_{0}, m, n\right)$. The NRMSD is approximately below $10 \%$ if the percentage of error is smaller than $20 \%$ for $\alpha, \beta, \tau$. It is interesting to observe that $m_{0}$ of I-mode is very robust to errors. This is due to the power consumption model, i.e., to the dominant factor $m_{0}$ of power consumption in I-mode. The robustness of MAC parameter is $m_{0}>n>m$ and $n>m>m_{0}$ for I-mode and $S$-mode, respectively. We can show that errors below $20 \%$ in the estimation of $\alpha, \beta, \tau$ give a performance degradation below $3 \%$ in terms of reliability, packet delay and energy gain for low traffic load.

\section{CONCLUSIONS}

In this paper we presented an adaptive medium access control (MAC) algorithm for minimizing the power consumption while guaranteeing reliability and delay constraints of the IEEE 802.15.4 protocol. The algorithm does not require any modifications to the IEEE standard. The adaptive algorithm is grounded on an optimization problem where the objective function is the total power consumption, subject to constraints of reliability and delay of the packet delivery and the decision variables are the MAC parameters (macMinBE, macMaxCSMABackoffs, macMaxFrameRetries) of the standard. The proposed adaptive MAC algorithm is easily implementable on sensor nodes by estimating the busy channel and channel access probabilities.

We investigated the performance of our algorithm under both stationary and transient conditions. Numerical results showed that the proposed scheme is efficient and ensures a longer lifetime of the network. In addition, we showed that, even if the number of active nodes, traffic configuration and application constrains change sharply, our algorithm allows the system to recover quickly and operate at its optimal parameter by estimating just the busy channel and channel access probabilities. We also investigated the robustness of the protocol to possible errors during the estimation process on number of nodes and traffic load. Results indicated that the protocol reacts promptly to erroneous estimations.

Future investigations include the use of the aforementioned achievements to the practical implementation on sensor nodes based on specific application constraints. In addition, we plan to extend our study to the IEEE 802.11 standard.

\section{REFERENCES}

[1] IEEE Std 802.15.4, September, Part 15.4: Wireless Medium Access Control (MAC) and Physical Layer (PHY) Specifications for Low-Rate Wireless Personal Area Networks (WPANs), IEEE, 2006. [Online]. Available: http://www.ieee802.org/15

[2] A. Willig, K. Matheus, and A. Wolisz, "Wireless technology in industrial networks," Proceedings of the IEEE, pp. 1130-1151, 2005

[3] P. Park, "Protocol design for control applications using wireless sensor networks," Royal Institute of Technology (KTH), Tech. Rep. TRITA-EE 2009:041, Oct. 2009, licentiate thesis.

[4] T. Abdelzaher, T. He, and J. Stankovic, "Feedback control of data aggregation in sensor networks," in IEEE CDC, December 2004

[5] S. Pollin, M. Ergen, S. C. Ergen, B. Bougard, L. Perre, I. Moerman, A. Bahai, P. Varaiya, and F. Catthoor, "Performance analysis of slotted carrier sense IEEE 802.15.4 medium access layer," IEEE Transactions on Wireless Communication, vol. 7, no. 9, pp. 3359-3371, 2008.

[6] M. J. Miller, C. Sengul, and I. Gupta, "Exploring the energy-latency trade-off for broadcasts in energy-saving sensor networks," in IEEE ICDCS, 2005.

[7] J. R. Moyne and D. M. Tilbury, "The emergence of industrial control networks for manufacturing control, diagnostics, and safety data," Proceedings of the IEEE, pp. 29 - 47, 2007.

[8] W. Zhang, M. S. Braniky, and S. M. Phillips, "Stability of networked control systems," IEEE Control Systems Magazine, pp. 84 - 99, 2001.

[9] IEEE Std 802.11 Wireless LAN Medium Access Control (MAC) and Physical Layer (PHY) Specifications, IEEE, 1999. [Online]. Available: http://www.ieee802.org/11

[10] F. Cali, M. Conti, and E. Gregori, "IEEE 802.11 protocol: design and performance evaluation of an adaptive backoff mechanism," IEEE Journal on Selected Areas in Communications, pp. 1774 - 1786, 2000.

[11] K. Yedavalli and B. Krishnamachari, "Enhancement of the IEEE 802.15.4 MAC protocol for scalable data collection in dense sensor networks," in ICST WiOPT, 2008.

[12] R. Bruno, M. Conti, and E. Gregori, "Optimization of efficiency and energy consumption in p-persistent CSMA-based wireless LANs," IEEE Transactions on Mobile Computing, vol. 1, pp. $10-31,2002$.

[13] V. Bharghavan, A. J. Demers, S. Shenker, and L. Zhang, "MACAW: A media access protocol for wireless LAN's," ACM SIGCOMM, 1994.

[14] B. Bensaou, Y. Wang, and C. C. Ko, "Fair medium access in 802.11 based wireless ad-hoc networks," in ACM MobiHoc, 
2000.

[15] Q. Pang, S. C. Liew, J. Y. B. Lee, and V. C. M. Leung, "Performance evaluation of an adaptive backoff scheme for WLAN: Research articles," Wirel.Commun. Mob. Comput., vol. 4, pp. 867-879, 2004.

[16] J. G. Ko, Y. H. Cho, and H. Kim, "Performance evaluation of IEEE 802.15.4 MAC with different backoff ranges in wireless sensor networks," in IEEE ICCS, 2006.

[17] A. C. Pang and H. W. Tseng, "Dynamic backoff for wireless personal networks," in IEEE GLOBECOM, 2004.

[18] P. Park, P. D. Marco, P. Soldati, C. Fischione, and K. H. Johansson, "A generalized markov chain model for effective analysis of slotted IEEE 802.15.4," in IEEE MASS, 2009.

[19] G. Bianchi, "Performance analysis of the IEEE 802.11 distributed cordination function," IEEE Journal on Selected Areas in Communications, vol. 18, pp. 535 - 547, March 2000.

[20] S. Coleri-Ergen, P. D. Marco, and C. Fischione, "MAC protocol engine for sensor networks," in IEEE Globecom, 2009.

[21] P. Park, P. D. Marco, C. Fischione, and K. H. Johansson, "Adaptive IEEE 802.15.4 protocol for reliable and timely communnicaions," KTH, Tech. Rep. TRITA-EE 2009:054, 2009.

[22] D. P. Bertsekas and J. N. Tsitsiklis, Parallel and Distributed Computation: Numerical Methods. Athena Scientific, 1997.

[23] A. Speranzon, C. Fischione, and K. H. Johansson, "Distributed and collaborative estimation over wireless sensor networks," in IEEE Conference on Decision and Control, 2006.

[24] C. Fischione, A. Speranzon, K. H. Johansson, and A. Sangiovanni-Vincentelli, "Peer-to-peer estimation over wireless sensor networks via Lipschitz optimization," in ACM/IEEE IPSN, 2009.

[25] F. Cali, M. Conti, and E. Gregori, "Dynamic tuning of the IEEE 802.11 protocol to achieve a theoretical throughput limit," IEEE/ACM Transactions on Networking, vol. 8, pp. 785 - 799, 2006.

[26] R. Jain, D. Chiu, and W. Hawe, "A quantitative measure of fairness and discrimination for resource allocation in shared computer systems," Digital Equipment Corporation, Tech. Rep., 1984. 